\title{
1 \\ Towards Computer-Aided Rapid Product Realization
}

\author{
G. J. Olling
}

Chrysler Corporation

800 Chrysler Drive CIMS: 483-05-01

Auburn Hills, MI 48326 USA

Tel: (248) 576-2788 Fax: (248) 576-2029

\section{Extended Abstract}

During the closing of the twentieth century, U.S. based manufacturers are contending with an increasing number of international competitors. In pursuit of world-class manufacturing status, U.S. firms have come to realize that corporate commitment to quality and responsiveness to customers' demands are now the benchmarks of outstanding enterprises. Industry leaders distinguish themselves from competitors by the rapidity of their product introductions, striving for the highest possible quality at the lowest possible price. World-class manufacturing has become a continual improvement process focusing on the need to reduce lead time dramatically while continuing to innovate and assure quality.

In this climate of intensified global competition, the computer technology of manufacturing has flourished. Designers and engineers now use Computer-Aided Design and Engineering ( $\mathrm{CAD} / \mathrm{CAE})$ tools to create three-dimensional images that can be shaded, analyzed, and optimized to refine the product design. Manufacturing engineers use Computer-Aided Manufacturing (CAM) systems for process planning, tool design, and machine programming. Administrators draw upon Management Information Systems (MIS) in production planning, production and inventory control, sales, research, and forecasting.

Computer-Integrated Manufacturing (CIM) is the ultimate goal in integrating $\mathrm{CAD} / \mathrm{CAE}$, CAM, and MIS technologies. However, the rapid development of these technologies has stretched and distorted the meaning of CIM. Today, the concept has elements of Group Technology (GT), Materials Requirements or Resource Planning (MRP), and ComputerAssisted Process Planning (CAPP). It also draws upon the fields of product design, production planning, and production control. In its implementation, CIM has been expressed in a tremendous number of specialized software packages developed by vendors with divergent perceptions of manufacturing and no great desire to integrate with on another. 
The need to stay abreast of product and process innovation worldwide and to build a world-class manufacturing operation will require the creation of industrial systems that convey the states of markets, competition, technology and the ensemble of techniques and technologies that automate, integrate, and optimize the process of manufacturing, from innovation to customer response.

Since prevailing need will be for rapid adjustment to changing demand, the systems approach requires an organizational framework that is flexible and adaptive, employees at all levels that are knowledgeable about human relations, methods, tools, and the effective use of existing technologies.

The successful enterprise of the 21 st century will be characterized by an organizational structure that efficiently responds to customer demands and changing global circumstances, a corporate culture that empowers employees at all levels and encourages constant communication among related groups, and a technological infrastructure that fully supports process improvement and integration. In changing itself to keep abreast of the broader transformation in manufacturing, the enterprise must look first at its organization and culture, and thereafter at supporting technologies. Too often, today, companies look for a quick fix of technology, throwing vast capital at its problems, only to find that its organization is still adhering to the same attitudes, methods, and schedules that caused the problems in the first place.

In the effort to achieve end-to-end integration, from the beginning of design to the completion of manufacturing, $\mathrm{CAD}$ data represents a critical resource. The ideal system would assure the integrity of $\mathrm{CAD}$ data by having everyone use the same integrated set of $\mathrm{CAD} / \mathrm{CAE} / \mathrm{CAM}$ tools, communicate over a single network, share a common database, and adhere to standard design practices.

The chief advantages of this uniform system would be to prevent the loss of design intent through the imperfect translation of data between systems, to eliminate the preparation of data before translation and the cleanup afterwards, and facilitate the implementation of concurrent engineering in product development and manufacturing divisions.

At Chrysler Corporation for example, we are pushing for a core-system solution to tighten the integration within the company and its many suppliers. A major concern is to minimize translation. Since competing CAD/CAM systems usually have differing approaches to data representation, translations between systems make arbitrary interpretations and compromises. In sensitive areas, such as the design of outer skins in automotive applications, translations do not preserve the original design intent and integrity well enough to produce a satisfactory product. Ultimately, the company would like to eliminate all intermediate forms, -- not only models from other CAD systems and the IGES files used to translate them, but physical bucks and mock-ups, clay models and background $\mathrm{CAD}$ models. Experience has shown that intermediate forms add delay and cost, create synchronization problems and confusion and lead to quality shortcomings. 
A video is presented to illustrate the application of $\mathrm{CAE} / \mathrm{CAD} / \mathrm{CAM}$ technology throughout the entire vehicle development process, starting with Conceptual Design, spanning Body Engineering, Digital Model Assembly, Powertrain Design, Rapid Prototyping, and finishing with Stamping and Assembly.

With a CAD/CAM foundation in place, Chrysler's next challenge is to incorporate business, engineering, manufacturing and suppliers know-how into a single integrated system. A "next-generation" CAD/CAM system will be based on precise solids with a feature-based structure that stores information related to all the steps in the product development and manufacturing processes. The system will have parametric associativity so that design changes in one aspect of the product are automatically reflected in all other aspects of the product. Finally, the system will be generative. Through built-in intelligence, engineering analysis models, manufacturing process models, business cost models, and purchasing materials models will all be generated from the base product design model. This kind of generation will be possible because the product model will no longer be geometry represented in the computer but rather a section of the on-line product database.

Although Chrysler has made steady progress in the evolution of its products and process development technology, it has much work planned for future development. The goal is to achieve barrier-free, real-time access to shared, associative product development information throughout the enterprise. Perhaps the most important lesson Chrysler has learned in its transformation is that corporate-wide integration and simultaneous engineering are not objectives that a single company, even a large one, can accomplish alone. Such profound changes require broad cooperation among the company, its design and component suppliers, its software and services vendors, educators and researchers in nearby communities and at-large, its customer groups and related professional organizations.

CAE, CAD, CAM, CIM, Product Realization

\section{Key Words}

